\title{
NEUE WIRTSCHAFTSGEOGRAPHISCHE KARTEN DES SCHWEIZERISCHEN MITTELSCHULATLASSES
}

\author{
Von Josr HösLI
}

Mehr als je rückt die wirtschaftsgeographische Betrachtung von Landschaften, Ländern, Ländergruppen und Kontinenten im Rahmen eines fortschrittlichen und aktuellen Erdkundeunterrichtes in den Vordergrund. In keinem Belange geographischen Arbeitens, das den Menschen als Gestalter der Landschaft zu würdigen versucht, kann der Gedankengänge des jungen, erst zu Ende des letzten Jahrhunderts aus der Praxis geborenen Zweiges der geographischen Wissenschaft entbehrt werden. So wachsen begreiflicherweise die Anforderungen an ein dem Unterricht zweckdienliches Anschauungs- und Werkmaterial. In erster Linie gelten sie der Karte, dem unentbehrlichsten und vornehmsten Hilfsmittel der geographischen Schulung. Tatsächlich ist das Verlangen nach zeitgemäßen Darstellungen groß. Ihm möchte die eben erschienene Jubilä umsausgabe des zum nationalen Lehrmittel gewordenen Schweizerischen Mittelschulatlasses besondere Rechnung tragen. Unter der Leitung des Redaktors ED. IMrof, Professor für Kartographie an der ETH., unter Mitarbeit von Prof. Dr. $\mathrm{H}$. Bossch, Ordinarius für Geographie und Direktor des Geographischen Institutes der Universität Zürich, und unter zeitweiliger Mithilfe der Herren cand. phil. WAITER FreI und .Gerhard StMmen haben sämtliche wirtschaftsgeographischen Karten durch den Verfasser mancherlei Umgestaltungen erfahren. Ihnen mögen einige Begleitworte allgemeinen und speziellen Inhaltes gewidmet sein.

\section{DIE STOFFLICHEN GRUNDLAGEN DER KARTENGEBUNG}

Wie jede Kartengebung, so gründet auch das Schaffen von wirtschaftsgeographischen Karten auf den unerläßlichen Grundlagen des darzustellenden Stoffes und der ihn darstellenden Mittel. Die Wahl der Projektion zur Kartenunterlage und ebenso die graphischen Möglichkeiten der Darstellung bieten heutzutage bei hochentwickelter Kartographie und Graphik keine ernsten Schwierigkeiten. Bedeutend größere, nicht selten unüberwindbare Schranken ergeben sich aus dem Mangel an Literatur, der für gewisse Länder aus verschiedenen Gründen bezeichnend ist. Immer wird eine gewisse unterschiedliche Gründlichkeit der sachlichen Darbietung auf die ungleichen Voraussetzungen in der Grundlagenbeschaffung zurückzuführen sein. Als weitere Imponderabilien türmen sich die Schwierigkeiten, die aus den stofflichen und zeitlichen Unterschieden des verfügbaren Materials resultieren. Was für ein Land vorhanden ist, fehlt für ein anderes; über dieses berichten neuere Werke, von jenem zeugen nur altersgraue Folianten. Und das Neueste steht zumeist aus, das Neueste des Wirtschaftsgeschehens, das wie kein anderes Gebiet menschlicher Kulturtätigkeit mannigfachen Wandlungen unterworfen ist. Im Zeitalter weltumspannender Wirtschaftspolitik hat wenig Bestand; gar vieles ist ständig in Fluß. Wissenschaft und Technik leisten den wachsenden und sich oft vielfältig ändernden Bedürfnissen mächtig Vorschub. Was soll mit den zu schaffenden Karten werden? Hüte man sich vor unüberlegten Forderungen an Darstellungen, die das Wirtschaftsleben größerer Erdräume festhalten sollen. Es lassen sich kaum mehr als Durchschnittsbilder für gewisse Zeitspannen schaffen, Durchschnittsbilder, die nur mehr oder weniger der Gegenwart entsprechen können. In Anbetracht dessen verliert die Diskussion um den schlecht oder gut gewählten Zeitpunkt der in den Jahren 1945 und 1946 erfolgten Revision der wirtschaftsgeographischen Atlaskarten von selbst an 
Kraft. Stofflich handelte es sich dabei lediglich um ein Nachführen der wesentlichen Veränderungen, die erstens zu Zeiten der vorletzten grundlegenden Umgestaltung des Lehrmittels (1928-1932) noch nicht bekannt waren und die zweitens das vergangene Jahrzehnt bis zum Vorkriegsjahr 1939 kennzeichnen ${ }^{1}$. Die derzeitigen, durch den zweiten Weltkrieg schlagartig ausgelösten Wandlungen zu erfassen, das muß einer zukünftigen Bearbeitung überbunden werden, die sich auf sorgfältige Grundlagen der Kriegsund Nachkriegszeiten stützen kann.

\section{DIE METHODISCHEN KRITERIEN}

Einmal über die mühsame bibliographische Sammeltätigkeit hinausgekommen, öffnet sich das weite Feld der Fragen nach den Ansprüchen des Unterrichtes, nach dem Weg der rechten Wissens- und Erkenntnisbildung wirtschaftsgeographischer Werte. So gesellen sich zu den Hindernissen und Schwierigkeiten der Grundlagenbeschaffung die wegweisenden Schranken des methodischen Vorgehens, dessen Unterlagen teils in den Gesetzen des Seelenlebens, teils im Wesen des Unterrichtsstoffes gegeben sind. Im Rahmen des materiell Möglichen bestimmen vor allem drei Kriterien das zu schaffende Kartenbild, das Was und Wie der vom Lehrer zu übermittelnden und vom Schüler zu apperzipierenden Darstellung. Es sind dies die Prinzipien der Stoffauswahl, der Anschaulichkeit und der Anschauung im tieferen Sinne des Wortes, deren Forderungen sich alle gleichzeitig geltend machen.

1. Die Auswahl des Stoffes hat sich nach der Zielsetzung des Unterrichtes zu richten, die, soll sie weise sein, der geistigen Auffassungskraft der verschiedenen Altersstufen zu entsprechen hat. Angesichts der weitgespannten Ansprüche an den Mittelschulatlas von seiten verschiedenartiger Schultypen (Gymnasium, Oberreal-, Handelsund kaufmännische Schule) und Altersklassen (12-18 und mehr Jahre) ist aber zu begreifen, daß eine weitgehende Beschränkung in der Auswahl nicht in Frage kommen kann. Der Atlas hat "Mädchen» für alle zu sein; und allen recht zu tun, verlangt immer vermittelnde Lösungen. Den im Lehrplan umschriebenen Bedürfnissen der unterschiedlichen Schulrichtungen und Schulklassen und zugleich den vielgestaltigen Forderungen des einzelnen Lehrers kann nur durch eine wissenschaftlich objektive Aufführung der wesentlichen Erscheinungen Genüge getan werden. Deshalb entbehren die Atlaskarten des nicht selten gewünschten großzügigen, verallgemeinernden Schematismus. Die Herstellung von Faustskizzen kann nie eine Angelegenheit des Mittelschulatlasses sein; dies wird immer dem Lehrer überlassen sein.

2. Auch dem Fragenkomplex nach der äußeren, graphischen Form des Darzustellenden ist mehr als früher Beachtung geschenkt worden, wodurch die Kartenzeichnung an Anschaulichkeit gewonnen haben mag. Eine sinngemäße und konsequent durchgeführte Farbgebung und Anwendung von Signaturen schaffen ein leichter lesbares Kartenbild. Die Wahl der Farben hat sich vor allem nach einer Farbenlogik zu richten, die ein Einfühlen und Eindenken in die dem Flächenkolorit zugrunde liegende Materie durch Assoziation erleichtert. Die detaillierte Führung der die Flächen gleichen Inhaltes umgrenzenden Linien soll ein mit den tatsächlichen Verhältnissen möglichst übereinstimmendes Bild ergeben, wodurch die Karte eine der topographischen Gestaltung entsprechende Plastik gewinnt.

1 Zur Illustration seien zufällig die Schwerpunktsverlagerungen in der Erdölwirtschaft, das Aufblühen der jungen Aluminiumindustrie und die moderne Entwicklung des kolonialen Landbaues im tropischen Afrika erwähnt. Es wäre wohl dankbar, dem Reichtum an Beispielen von wirtschaftlichen Wandlungen nachzugehen, die seit den zwanziger Jahren das Wirtschaftsgefüge mancher Länder bereichern und die ihrer Bedeutung wegen in den neu ausgeführten Karten entsprechenden Niederschlag finden mußten. Doch bedürfte dies einer besonderen, umfangreichen Arbeit. 
3. Methodisch ungleich komplizierter ist die Verwirklichung des Prinzipes der Anschauung, der Schaffung deutlicher Vorstellungen und Erkenntnisse, der Begriffsbildung. Die innere Form der Darstellungen, vom Stand der Lehrmeinungen über die Aufgaben der Geographie beeinflußt, hat sich weitgehend den Stufen der im Schüler entwickelnden Auffassungskraft anzupassen. Wie bei der Stoffauswahl, so müssen aber auch hierbei weitreichende Konzessionen an die verschiedenartigen Ansprüche gemacht werden. Diese äußern sich in der bunten, vielseitigen Folge von Karten bald einfacheren, bald schwereren Inhaltes, von Karten, die einmal ein bloßes Inventar von Einzelerscheinungen bedeuten, dann aber wieder begriffliches Denken voraussetzen. So wie in der Reihenfolge der Atlaskarten kein bestimmtes Programm der Stoffbehandlung berücksichtigt werden kann, ebensowenig ist es möglich, daß ein ganz bestimmtes methodisches Vorgehen Richtschnur für die Art und Weise des darzustellenden Inhaltes sein kann.

\section{DIE ART DER WIRTSCHAFTSGEOGRAPHISCHEN ATLASKARTEN}

Nach den mehr grundsätzlichen Bemerkungen zur Schaffung der wirtschaftsgeographischen Atlaskarten, die den stoffgegebenen Möglichkeiten und den praktischen Forderungen des Unterrichtes gegolten haben, soll es nicht unterlassen bleiben, auf die verschiedenen Arten der dem Inhalt nach nicht näher zu beschreibenden Kartenbilder einzugehen.

1. Analytische Darstellungen. Eine Mehrzahl der im Atlas enthaltenen Karten beschränkt sich auf Einzelobjekte des Wirtschaftslebens. Es sind analytische Darstellungen, eigentliche Spezialkarten, die einen bestimmten Wirtschaftsgegenstand, zumeist aber mehrere nebeneinander auf Kartenunterlagen in irgendwelcher Form vermerken. Diese Darstellungsart gilt als die älteste und wurde früher ausschließlich von Geographen verwendet. Heutzutage ist sie weder ein Privileg des Geographen noch seiner Wissenschaft spezifisch. Als "kartographische Methode» ist sie schon lange wissenschaftliches und populäres Allgemeingut geworden.

Die einfachsten, zugleich ältesten analytischen Wirtschaftskarten sind bloße Verbreitungskarte $n^{2}$. Sie geben die örtliche Verteilung ausgewählter Wirtschaftsobjekte wieder, als Produktenkarten z. B. Produkte der Landwirtschaft, des Bergbaues und der Fischerei. Solche Karten liegen in mehr oder weniger reiner Form für fast alle Länder, Kontinente und als Erdübersichten vor. Wertvoll wären auch Karten, die nebst der örtlichen Verteilung, in Form von Kreisscheiben, Aufschluß über produzierte und konsumierte Quantitäten und Qualitäten geben. Ihrer Abhängigkeit von zeitlich sich verändernden statistischen Werten wegen wurde von ihnen Abstand genommen. Der zahlenmäßige Beleg bleibt dem Lehrer überbunden. Immerhin wurde z. B. für den Bergbau versucht - wenn dies auch nicht immer zum Ausdruck kommt -, die wesentlichsten Produktionsstätten entweder durch größere Signaturen oder durch eine Anhäufung von solchen $\mathrm{zu}$ betonen ${ }^{3}$.

2. Synthetische Darstellungen. Das Ziel der eigentlichen, vollwertigen wirtschaftsgeographischen Karte gilt aber der synthetischen Darstellung, die gewisse, nach Umfang und Inhalt bestimmte Einheiten aufstellt, mit denen gleich eine ganze Reihe von Vorstellungskomplexen zusammengefaßt wird. In dieser Richtung bedeuten die

${ }^{2}$ Eine der ältesten Produktenkatte stammt vom Geographen RIrrer aus dem Jahre 1845 und veranschaulicht die "Verbreitungssphäre des Zuckerrohrs».

${ }^{3}$ Viele in den Entwürfen nach statistischen Werten der Produktion gemachte Unterschiede sind infolge der notwendigen starken Bildverkleinerung der generalisierenden Hand des Graphikers zum Opfer gefallen. 
meisten Karten einen neuën Versuch, der die ehemaligen, àm besten in ihrer Legende -als wenig befriedigend zu erkennenden Darstellungen ersetzt.

3. Kombinierende Darstellungen. Bevor auf die Besonderheiten der neu geschaffenen synthetischen Karten eingegangen werden soll, möchte noch die dritte Gruppe "von Atlaskarten Erwähnung finden, da analytische Betrachtungsweise sich mit synthetischer Darstellung vermengt.

\section{DIE NEUEN KARTEN DER WIRTSCHAFTSLANDSCHAFTEN}

Die für die Erdteile und die Weltübersicht neugestalteten Karten synthetischer Art gründen auf einer in den Jahren 1944 und 1945 als Assistenzarbeit unter Leitung von Prof. Dr. H. BozsCH entworfenen Originalwandkarte der Wirtschaftslandschaften der Erde, deren Inhalt mit einigen Abänderungen und Nachträgen übernommen wurde. Zu ihrem Verständnis sei ein Abriß älterer Versuche gegeben.

\section{1. Ältere Versuche}

Im Vordergrund älterer synthetischer Darstellungen steht die bis anhin bekannteste und immer noch viel verwendete Schulwandkarte von Wilhelm Osbahr (3. Auflage, 1925). Osbarr verwendet als synthetische Einheiten die Landbauzonen ENGELBREChTs, eine zonale Gliederung, die auf einer im großen ganzen symmetrischen Anordnung der Klimagürtel beruht ${ }^{4}$. Demnach teilt er die produktiven Gebiete in sechs Kulturzonen auf: in den äußern und innern Getreidegürtel des gemäßigt warmen Klimabereiches, in die äußere und innere Zone der Getreide- und Genußpflanzen der Subtropen und ebenso in eine äußere und innere tropische Zone der Getreide-, Genuß- und Industriepflanzen. Jede dieser Zonen wird mit einem besonderen Farbton gekennzeichnet. Die einzelinen wichtigen Produkte sind im Sinne einer kombinierenden Karte durch Zeichen; Buchstaben und Wörter hervorgehoben. Gemäß dem damaligen Ziel der Wirtschaftsgeographie, die in erster Linie dem Einfluß der Physis der Wirtschaftsräume auf die Gütererzeugung und Güterbewegung nachspürte, bezeichnet PARAvicINI die Karte von OSBAHR in einer ausführlichen Rezension als die wirtschaftsgeographische Weltkarte, im Gegensatz zu derjenigen von LANGHANS, welche «die physikalisch-geographischen Grundlagen völlig vernachlässigt $)^{5}$.

Unberǘcksichtigt aber bleibt bei OsBaHR der Mensch als Gestalter und Träger der Wirtschaft, obwohl schon seit der Jahrhundertwende namhafte Gèographen bei der Betrachtung der Beziehungen zwischen Natur und Mensch das Hauptgewicht auf den Menschen legen ${ }^{6}$. In diesem Zusammenhang muß die 1903 erschienene Karte von FRIEDRICH erwähnt werden, der als besondere Aufgabe wirtschaftsgeographischer Karten die Darstellung von Wirtschaftsstufen postulierte. Als Einteilungsprinzip gilt ihm die psychische Stellung des Menschen gegenüber der Natur, das Maß der Befreiung seiner Bedürfnisbefriedigung vom Naturzwang. Die Bedeutung seiner vier Wirtschaftsstufen - die der tierischen Wirtschaft oder des Reflexes, des Instinktes, der Tradition und der Wissenschaft - sieht er darin, daß . sie erstens die geschichtliche Entwicklung erkennen lassen, woraus die "Tendenz des wirtschaftlichen

4 Engelbrecht, Th.: Die Landbauzonen der Erde. Ergänzungsheft Nr. 209 zu Petermanns Mitteilungen (H. Wagners Gedächtnisschrift). Gotha 1930.

5 Paravicini, E.: Wilhelm Osbahrs Karte zur Wirtschaftsgeographie der Welt und die Prinzipien der kartographischen Darstellung der Weltwirtschaft. Siehe Abdruck der Schweizerischen Handelswissenschaftlichen Zeitschrift 1926.

- SCHLÜTER schreibt 1907: «Erste Voraussetzung ist immer der Mensch selbst mit seiner physischen und psychischen Organisation.» Hetrner betont im selben Jahre: "Bei der Betrachtungsweise, daß die Naturbedingung Grundlage des menschlichen Wirkens sei, kann man überhaupt nur zu Möglichkeiten kommen.» Der tatsächliche Zustand aber "geht aus dem Wollen und Können der Bevölkerung tiervor». KRAus fordert schon 1903 eine "Vergeistigung des der Wirtschaftsgeographie von allen.Seiten zuströmenden Materials durch eine vergleichende Betrachtung ihrer Erscheinungen über das Erdganze hin, unter dem Gesichtspunkt der Entwicklung».

SchlüreR, O.: Uber das Verhältnis von Natur und Mensch in der Anthropogeographie. Verhandlungen des 16. deutschen Geographentages, Berlin 1907, S. 305; Hetrner, A.: Die Geographie des Menschen; ebenda, S. 288; KrAus, A.: Geschichte der Handels- und Wirtschaftsgeographie, Verhandlungen des 14. deutschen Geographentages, Berlin 1903, S. 111. 
Fortschrittes erhelle» und zweitens die «heutige Wirtschaft der Exde nach der Höhe zu klassifizieren erlauben»?.

Von besonderer Wichtigkeit ist die noch ältere Karte der Wirtschaftsformen vom Ethnographen HaHN (1892) ${ }^{8}$. HaHN unterscheidet als solche die der Jäger und Fischer, des Hackbaues, des Plantagenbaues, des europäisch-westasiatischen Ackerbaues, der Viehwirtschaft und des Gartenbaues. Diese Wirtschaftsformen bedeuten ihm kulturelle Einheiten, die das Wirtschaftsleben der betreffenden Völker eindeutig prägen. Ihrer bedienen sich in irgendeiner Art und Weise die meisten der moderneren Darstellungen. SAPPER legt seiner "wirtschaftlichen Ausnützungskarte der Erde» acht Wirtschaftsformen zugrunde. Er erweitert die Zahl der von HaHN aufgestellten Einheiten um die Formen des Pflanzstockund Grabstockbaues, die er vom eigentlichen Hackbau abtrennt ${ }^{9}$. Der Amerikaner WhITrLESEY gliedert in einer 1936 erschienenen Arbeit die Erde in elf Landwirtschaftsregionen auf ${ }^{10}$.

\section{Die moderne wirtschaftsgeographische Zielsetzung}

Grundlegende wirtschaftsgeographische Bedeutung erhielt der von HaHN erstmals geprägte Begriff der Wirtschaftsform durch den noch im Fluß befindlichen Wandel geographischer Zielsetzung, womit selbstverständlich auch die Fragestellung und der Aufgabenkreis der Wirtschaftsgeographie verknüpft ist. Betrachtete man noch vor fünfzehn Jahren die Beziehungen zwischen dem wirtschaftenden Menschen und der bewirtschafteten Erde als spezifischen Gegenstand wirtschaftsgeographischer Forschung, so wird heute die Wirtschaftslandschaft als Gesamterscheinung untersucht. So steht z. B. nicht mehr die tropische Plantagenwirtschaft als solche und ihre Produktion im Vordergrund einer Untersuchung, sondern die Landschaft des Plantagenbaues mit all ihren Erscheinungen, die ursächlich damit verknüpft sind. Deshalb ist der Begriff der Wirtschaftsform, als die Art und Weise, wie der Mensch sich die Güter der Erde zu beschaffen und verwerten sucht, vom Wirtschaftsgeographen voll und ganz übernommen worden. WarBEL betont, daß eine begriffliche Unterscheidung von Wirtschaftsformen die wesentliche Grundlage der neuen Wirtschaftsgeographie sei ${ }^{11}$. Ein System derselben aber existiert noch nicht. WArBEL nennt als Beispiele von Wirtschaftsformen: den Pflanz- oder Hackbau, den ostasiatischen Gartenbau, den Pflugbau, Hirtennomadismus, die Weidewirtschaft und den Plantagenbau.

\section{Der methodische Aufbau der neuen Karten}

Auf dem soeben skizzierten Ziel der modernen Wirtschaftsgeographie gründen die neuen Karten. Sie streben eine durch die Zweckbestimmung als didaktische Hilfsmittel erwünschte übersichtliche, klare Gliederung in Typen von Wirtschaftslandschaften an. Diese unterscheiden sich mittels der Wirtschaftsform, der Art und Weise der wirtschaftlichen Nutzung also, dem wesentlichsten Merkmal der Wirtschaftslandschaft. Die Ausscheidung von Wirtschaftsformen erforderte eine gedankliche Hauptarbeit, die zum Teil in Anlehnung an die bereits genannten Arbeiten von HAFN und WhITTLESEY geschah. Als weiteres Merkmal zur Klassifizierung dient das Maß der weltwirtschaftlichen Verflechtung; zudem berücksichtigt sie auch die Intensität der Bewirtschaftung, die z. B. als Hauptmerkmal den einzigartigen Länderkarten des englischen Handelsatlases zugrunde liegt ${ }^{12}$. So werden extensive und intensive Formen unterschieden. Die Vielzahl der Wirtschaftsformen - es sind ihrer 18 - kann übersichtsmäßig zu folgenden Gruppen zusammengefaßt werden:

7 FRIEDRICH, E.: Einige kartographische Aufgaben in der Wirtschaftsgeographie. Verhandlungen des 14. deutschen Geographentages, Berlin 1903, S. 112-125.

8 Hahi, E.: Die Wirtschaftsformen der Erde. Petermarins Mitteilungen 1892.

- SAPPER, K.: Allgemeine Wirtschafts- und Verkehrsgeographie. Leipzig 1930. S. 133.

10 Whitrlesey, D.: Major agricultural regions of the Earth. Ann. Assoc. Geographers 1936.

11 Waibel, Leo: Probleme der Landwirtschaftsgeographie. Breslau 1933.

12 Chambers of Commerce Atlas. London 1925. 
Die Typen der. Wirtschaftslandschaften

\begin{tabular}{lll} 
Wirtschaftsformen & \multicolumn{1}{c}{$\begin{array}{c}\text { Vorwiegende } \\
\text { Selbstversorgungswirtschaft }\end{array}$} & $\begin{array}{l}\text { Vorwiegende binnen- und weltmarkt- } \\
\text { orientierte Wirtschaft }\end{array}$ \\
\hline $\begin{array}{l}\text { Okkupations- und } \\
\text { Waldwirtschaft }\end{array}$ & $\begin{array}{l}\text { Sammel-und Rodungswirtschaft } \\
\text { der Naturvölker, Jagd und Fischfang }\end{array}$ & $\begin{array}{l}\text { Sammel-und Rodungswirtschaft } \\
\text { der Kulturvölker, Jagd, Fischfang } \\
\text { Holzwirtschaft }\end{array}$ \\
\hline $\begin{array}{l}\text { Reine } \\
\text { Viehwirtschaft }\end{array}$ & Nomadische Viehwirtschaft & $\begin{array}{l}\text { Vieh-Weide-Wirtschaft } \\
\text { (Farmbetrieb) } \\
\end{array}$ \\
& & $\begin{array}{l}\text { 1. vorwiegend Schafzucht } \\
\text { 2. vorwiegend Rinderzucht }\end{array}$ \\
\hline
\end{tabular}

\begin{tabular}{ll}
\hline Primitiver Ackerbau & ViehwirtschaftderEingeborenen \\
(Wander-Feldbau) & mit Feldbau (Hacke, Grab- und \\
mit Viehwirtschaft & Pflanzstock) \\
& Feldbau der Eingeborenen mit \\
& geringer Viehhaltung
\end{tabular}

\begin{tabular}{lll}
\hline $\begin{array}{l}\text { Pflugbau } \\
\text { mit Viehwirtschaft }\end{array}$ & $\begin{array}{l}\text { Extensiver Landbau } \\
\text { (inkl. Pionierwirtschaft) } \\
\text { Intensiver Landbau ohne Reis } \\
\text { Intensiver Landbau mit Reis } \\
\text { (Gartenbau) }\end{array}$ & $\begin{array}{l}\text { Getreidewirtschaft } \\
\text { Mediterrane Landwirtschaft } \\
\text { Gras-Milch-Wirtschaft }\end{array}$ \\
& $\begin{array}{l}\text { Intensiver Ackerbau mit inten- } \\
\text { siver Viehwirtschaft } \\
\text { Spezialkulturen: Gemüse, Früchte, } \\
\text { Wein }\end{array}$ \\
& $\begin{array}{l}\text { Plantagenwirtschaft } \\
\text { 1. Plantagenmäßiger Anbau der Ein- } \\
\text { geborenen im Dienste der Kultur- } \\
\text { völker }\end{array}$ \\
& $\begin{array}{l}\text { 2. Farm-, Pflanzungs- und Plantagen- } \\
\text { betriebe der Kulturvölker (inkl. } \\
\text { Baumwollgürtel der USA.) }\end{array}$
\end{tabular}

Industrielle.

Bergbau

Wirtschaft

Industrie

Nicht fehlen darf die Industrie, die, insofern sie eine großräumige, dichte Verteilung aufweist, mit dargestellt wird. Die Schwerindustrie findet besondere Erwähnung. Ferner werden die für die Weltwirtschaft wichtigen Fischereigebiete angedeutet. Für die Weltübersicht geben zudem die als blaue Bänder gezeichneten Seehandelswege ein Bild der durch den Bedarf verursachten Güterbewegung und lassen dadurch auch die großen Verbraucherzentren ahnen. Thre Breite steht in Beziehung zum gesamten Handelswert der 1937 in beiden Richtungen ausgetauschten Waren. Innerhalb der gesamten Bandbreite versucht ein weiterer Bandstreifen - der dunkelblaue - im gleichen Sinne den Anteil der Fertigprodukte, der Manufakturwaren, auszuscheiden. Dadurch gelingt es ohne weiteres, den Überseehandel aller übrigen Waren: der Rohstoffe und Halbfabrikate, Lebensmittel, Getränke und lebenden Tiere, quantitativ abzuschätzen ${ }^{13}$.

\section{Stoffliche Grundlagen und graphische Ausführung}

Die Zahl der verarbeiteten Materialien ist Legion. Jegliche neuere Wirtschaftskarte, die zur Verfügung stand, wurde herbeigezogen. Große Schwierigkeiten bereiteten die nach den verschiedensten Gesichtspunkten behandelten Detaildarstellungen. Sie mußten oft mit Hilfe von Beschreibungen auf den grundlegenden Nenner gebracht werden.

Der Verlauf der Grenzlinien, welche die Wirtschaftslandschaftstypen abgrenzen, entspricht möglichst den wirklichen Verhältnissen. Für Überlagerungsgebiete zweier

13 Die Darstellung der Handelsbänder konnte Seminar-Arbeiten des Geographischen Institutes der Universität Zürich entnommen werden. 
Wirtschaftsformen hingegen ist eine signaturenhafte Abgrenzung unumgehbar. Das Großrelief, das schon durch die Anordnung der Typen zum Ausdruck gelangt, wird durch weiße Aussparungen betont, welche die unproduktiven Gebirgsöden wie auch die Wüsten aller Breiten wiedergeben.

Allgemein tragen die Wirtschaftsformen vorwiegender Selbstversorgung blasse, zurücktretende Farben, diejenigen vorwiegender Marktorientierung sind kräftiger koloriert. Farbpunkte in Farbflächen deuten in beiden Fällen, wo sie verwendet werden, Ähnlichkeiten. Die Farbschraffuren beschränken sich auf die Fälle gegenseitiger Durchdringung von Formtypen.

\section{Kurze Charakteristik der Legendeninhalte}

Zum besseren Verständnis der die Typen von Wirtschaftslandschaften prägenden Wirtschaftsformen diene folgender skizzenhafte Überblick.

Die mit der blassesten grünen Farbe gekennzeichneten Gebiete, die einen großen Teil verschiedener Kontinente einnehmen, geben eine Wirtschaftsform wieder, die als Sammel- und Rodungswirtschaft der Naturvölker angesprochen wird. Sie ist weder in ihren Kulturerscheinungen noch im wirtschaftslandschaftlichen Bild einheitlich, das sich übrigens fast völlig mit dem der Naturlandschaft deckt. So umfaßt ihr Typus die Wirtschaftslandschaften der sich vollständig selbstgenügenden Sammler und Jäger der tropischen Regenwälder, der südasiatischen Gebirgs- und Sumpfgebiete, der Tundren und subpolaren Nadelwälder, der wasserarmen Salz- und Wüstensteppen. Er enthält auch die Landschaften eines primitiven Ackerbaues in den tropischen Urwäldern, wo auf Rodungsinseln mit Pflanz-, Grabstock oder Hacke Wanderfeldbau betrieben wird. Wurzel- und Knollengewächse, tropische Baumfrüchte sind die wichtigsten Nahrungslieferanten. Hauptgebiet des Pflanzenstockbaues ist das tropische Amerika, dasjenige des Grabstockes das melanesisch-papuanische Kulturgebiet. Der Hackbau findet in Afrika seine größte Verbreitung.

Sammelwirtschaft und Jagd stehen aber auch im Dienste der Weltwirtschaft. In den tropischen Wäldern wird eine Vielzahl von pflanzlichen Produkten gesammelt, Edel- und Farbhölzern geschlagen: Kautschuk, Paranüsse und Vanille in den Flußniederungen des Amazonas, Kautschuk und Baumharze für die Herstellung von Lakken im Kongobecken; Mahagonihölzer stammen aus Westindien, Mittelamerika, Teakholz aus Siam und Burma, Blauholz und Chicle zur Kaugummifabrikation von der Halbinsel Yucatan. Der Chaco liefert Quebracho, Südbrasilien und Paraguay den von einer Stechpalmenart gewonnenen Mate.

Die subarktischen Waldländer und Tundren hingegen sind ihres Wildreichtums wegen bekannt. Kanada ist der wichtigste Pelzlieferant. Trapper und Jäger großer Gesellschaften durchschweifen die unermeßlichen Weiten. Ihre Forts und Handelsposten schieben sich Flußläufen entlang, immer weiter nordwärts.

Als besondere Form muß die intensive, oft planmäßige Nutzung der Waldgebiete, die skandinavische, russische, nordamerikanische, australische Holzwirtschaft festgehalten werden. Das Weichholz der Kiefern, Fichten, Tannen, Lärchen und Zedern geht als Brenn-, Bau- und Grubenholz in alle Welt und bietet den bedeutendsten Rohstoff für die Papierfabrikation.

Reine Viehwirtschaft finden wir in den Ländern, die zum Teil wegen ungenügender Sommerwärme, zum Teil wegen ungenügender Niederschläge für den Feldbau ungeeignet sind. Weltwirtschaftlich unbedeutend ist die Tierhaltung der Nomaden: die Renntiere der Eskimos, der Lappen, der sibirischen Völker - die Rinder-, Pferdeund Kamelherden der Wüsten- und Steppenbewohner.Afrikas und Asiens, die Schaf-, Ziegen- und Yakzucht der asiatischen Hochgebirgsländer. Sind doch die Motive ihrer 
Viehwirtschaft vielfach keine rein wirtschaftlichen und spielen die Tiere im Nahrungshaushalt dieser Naturvölker oft eine untergeordnete Rolle.

Größere Aufmerksamkeit erheischen die Landschaften der kommerziellen ViehWeide-Wirtschaft, die bald extensiv, bald intensiver, zumeist in Großfarmen betrieben wird. Als Beispiele seien genannt: die Steppen und Trockenwälder Argentiniens, Uruguays, von Brasilien und Paraguay, Australien und Neuseeland, der südafrikanischen Union, die Prärien, Gebirgs- und Plateauländer des vereinstaatlichen Westens. Sie versorgen die Welt ausschließlich-mit Wolle und Fellen, mit Fleisch und Fett.

Der primitive Ackerbau hat seine mächtigste Verbreitung in der Grasländern und Trockenwäldern Afrikas. In Brandkultur wird Gras, Busch und Wald gerodet und vorwiegend von den Frauen mit der Hacke zum Feld bestellt. Knollenfrüchte, Hirsearten und Mais sind die zur Selbstversorgung wichtigsten Anbaugewächse. Das Unkraut, der Ausschlag der im Boden verbliebenen Wurzeln und die Verarmung des Bodens zwingen, nach zwei bis dreijähriger Nutzung ein neues Bodenstück unter Wirtschaft zu nehmen (Wanderfeldbau oder Shifting cultivation). Nach der Bedeutung der diesem Feldbau zugesellten Viehzucht unterscheiden wir zwei Wirtschaftsformen:

1. den Feldbau mit unbedeutender Viehhaltung, in den konzentrisch um den Urwald sich anordnenden Feuchtsteppen, wo die Tsetseseuche die Großviehwirtschaft ausschließt;

2. die Viehwirtschaft mit Feldbau, in Gebieten der Trockensteppe, wo Viehzucht dem Ackerbau gleichwertig und übergeordnet erscheint. Die Kultur dieser Wirtschaftsform ist vielfach das Resultat gegenseitiger Durchdringung seßhafter Pflanzerbevölkerung mit nomadischem Rinderhirtentum.

Ähnliche Verhältnisse zeigen die Hochgebirgsregionen Süd- und Mittelamerikas. Auf den klimatisch gemäßigten bis kalten Hochflächen der Andenländer Peru und Bolivien ist die Zucht des Lamas und des Schafes nebst geringem und primitivem Anbau Grundlage der Volksernährung. In den nördlicheren Andengebieten hingegen dominiert der Feldbau.

Eine Vielzahl von landwirtschaftlichen Formen, unter ihnen die weltwirtschaftlich wichtigsten, vereinigt die Gruppe des stets von der Viehwirtschaft begleiteten höheren Ackerbaues, des Pflugbaues. Er hat sich den größten Teil des außertropischen, für Ackerbau geeigneten Gebietes erobert. Außerordentlich groß ist die Verschiedenheit der Bodenbearbeitung. Produktionsziel, Erträge, Betriebsform wie Betriebsgröße variieren stark.

Eine einzigartige Stellung nimmt der den Monsunländern und den alten Bewässerungskulturen Afrikas und Westasiens eigene intensive Landbau ein. Er erzielt durch sorgfältige Düngung und Bodenbearbeitung, vielfach auch durch künstliche Bewässerung, auf kleinsten, zudem stark parzellierten Betriebsflächen höchste Erträge. In erster Linie genügèn diese dem Eigenbedarf. Die Viehhaltung ist gering und nur insofern von wirtschaftlichem Wert, als sie zum Pflügen Zugkräfte liefert. Büffel und Rind sind die wichtigsten Zug- und Lasttiere. Milchgenuß ist unbekannt; religiöse Vorschriften verbieten das Schlachten. Größte Sorgfalt gilt dem Dünger. Als solche dienen in Ostasien die menschlichen Abfallstoffe, Abfälle der Seidenzucht, Kompost, Grünzeug u. a.m.

Der tierische Dünger spielt hier, im Gegensatz zur indischen Landwirtschaft, wo vor allem Rinderdung verwendet wird, keine Rolle. Mit Recht kann dieser Landbau als Gartenbau bezeichnet werden, dem Gemüsegartenbau der großen Stadtgebiete Europas vergleichbar. In vielen Fällen bedient sich der Bauer zwar nur der Hacke; doch findet der Pflug allgemeine Verwendung. 
Als besondere Form muß der durch den Reisbau gekennzeichnete intensive Landbau aufgeführt werden. Der Anbau des Reises, der Hauptspeise des Ost- und Südasiaten, prägt eindeutig das Bild jener Landschaften. Alles Land, soweit Bewässerung möglich ist, ist Reisland, das oft ohne Brache unter Kultur steht. Auf Trockenfeldern, die lediglich auf Regen angewiesen bleiben, gedeihen Weizen, Hirse, Mais, Baumwolle, Hackfrüchte und Gemüse. Burma, Siam und Indochina versorgen mit beträchtlichen Produktionsüberschüssen die alte Welt. Die Reisländer Indien, Java, China und Japan hingegen vermögen sich ihrer überaus dichten Bevölkerung wegen nicht selbst zu versorgen.

Die als Landbau ohne Reis genannte Wirtschaftsform umfaßt die Wirtschaftslandschaften der nordchinesischen Ebene, der Mandschurei, der obern Gangestalung, des Fünfstromlandes, das mesopotamische Zweistromland und die Niloase Ägyptens. Bewässerungskultur ist für den intensiven Anbau in den oft von Dürren bedrohten Ländern von hoher Bedeutung. Als Anbaupflanzen herrschen Weizen, Hirsearten, Baumwolle und Ölfrüchte vor.

Eine Fülle der verschiedenartigsten Wirtschaftslandschaften weisen den Typus eines mehr oder weniger extensiven Pflugbaues auf, der vorwiegend der Selbstversorgung dient. Nicht selten begleiten Holz- und Weidewirtschaft diese Wirtschaftsform. Als Beispiele seien erwähnt: die an Grundwasser und Flußzonen gebundenen Oasenlandschaften Afrikas und Asiens. Intensive Bewirtschaftung ist hiebei nicht selten, was des tüpfelartigen Ausmaßes wegen nicht ausgeschieden werden konnte. Ebenso inselartigen Charakter besitzen die Pionierlandschaften des nördlichen Nordamerika, diejenigen von Sibirien, wo die Getreidefelder noch oft nach altem Dreifeldersystem bebaut werden, auch die der südrussischen Steppen, wo Nomaden zum Feldbau übergegangen sind. Dazu gehören die mehr flächenhaft ausgedehnten Wirtschaftslandschaften des Dekkan, die Berg- und Hochländer von Abessinien, Mexiko, Nordafrika und Spanien, die Berggebiete verschiedener europäischer Staaten. Auffällig zeichnet sich diese Wirtschaftsform in einem breiten binnenländischen Gürtel, der sich von den Ostseestaaten über Großrußland bis nach Sibirien hineinzieht. Auf seiner Bodenwirtschaft lastet der Fluch des nährstoffarmen Podsols. Die ausgelaugten Böden bieten bei extensiver Bewirtschaftung nur minderwertige Ernten. Wiesen und Weiden treten stark in den Vordergrund.

Der in den Rahmen marktorientierter Wirtschaft eingespannte moderne Pflugbau prägt die entwickeltsten Wirtschaftslandschaften der subtropischen und gemäßigten Breiten. Sie sind bald einfacher, bald komplexer Struktur. Die nur durch die Industrialisierung verständliche straffe, kommerzielle und kapitalistische Wirtschaftsorganisation verleiht ihnen gemeinsame Züge. Die Erkenntnisse der Wissenschaft und die Werkzeuge der Technik, Rentabilitätsfragen und Wirtschaftspolitik beherrschen den Wirtschaftsprozeß: künstlicher Dünger, arbeitersparende Maschinen, wertvollstes Saatgut und vielseitige, rationelle Produktenverwertung charakterisieren ihre Betriebsformen.

Fast einheitliches Aussehen tragen die jungkolonisierten, dünnbesiedelten Ge tr e id elandschaften großräumiger Steppen und Prärien. Weizen ist fast ausschließliches Anbauprodukt. Monokultur. Großgrundbesitz und ebenes Gelände erleichtern die maschinelle Bestellung der monoton weiten Ackerfluren. Die Erträge sind im Vergleich zu denjenigen der Intensiv-Ackerbaugebiete gering; die Getreidewirtschaft zeigt durchaus extensiven Charakter. Dürren und Marktverhältnisse bedingen auffällige Produktionsschwankungen. Die Größe der Anbauflächen, zusammen mit der geringen Volkszahl, gestaltet diese Räume zu den bekannten Weizenkammern der Erde: z. B. die Prärien NW-Amerikas, die Pampas Argentiniens, SE- und W-Australien, die Schwarzerdesteppen der Sowjetunion.

Eine ganz andere Physiognomie besitzen die Landschaften der intensiven Mittelmeerwirtschaft. Als ein Saum, der sich an fruchtbare, bewässerte Ebenen und Fluß- 
täler hält, umzieht diese Wirtschaftsform das europäische Mittelmeer, zeichnet das Kapland und Mittelchile. Viehzucht ist allgemein üblich, doch von untergeordneter Bedeutung. Eigen ist dieser Form ein vielseitiger Ackerbau in Mischkulturen, die Polykultur. Feldfrüchte und Reben gedeihen in Kombination mit Fruchtbäumen. Sie dienen beidem: dem Eigenbedarf und dem Markt. Haine von Agrumen, Feigen und Datteln, Kastanien- und OOlbäumen liefern für den Regional- und Welthandel wertvolle Erzeugnisse.

Ähnlich und doch verschieden - weshalb sie als besonderer Typ aufgeführt werden muß - ist die Nutzungsform der Spezialkulturen: der USA., in Kalifornien, der atlantischen Küste; von Australien, in der Riverina, Wimmera, um Adelaide; des argentinischen "Kaliforniens» von Cordova, Mendoza, San Juan, San Rafael. Im subtropischen Bereich sind es zumeist Bewässerungslandschaften modernster Gestalt, wo die Markterzeugnisse der Mittelmeerwirtschaft räumlich gesonderten, plantagenmäBigen und rationellsten Anbau erfahren. In erster Linie wird für die Ausfuhr produziert. Besondere Erwähnung fordert der vereinstaatliche Gemüsegürtel des nord- und mittelatlantischen Küstensaumes.

Die Landschaften vorwiegender Gras- und Milchwirtschaft, die zum Teil ihre Molkerei- und Fleischprodukte auf den Weltmarkt bringen, beschränken sich zur Hauptsache auf die USA., West-, Mittel- und Nordeuropa, Australien und Neuseeland.

Größere Verbreitung zeigt der intensive Ackerbau, der Binnen- und Weltmarktbedeutung besitzt. Ihm parallel geht eine intensive Viehzucht. Der Mais- und Mastviehgürtel der USA. findet ein Ebenbild in den Pampas von Argentinien. Kleineren Raumes sind das "Maisdreieck» der südafrikanischen Union, die Mischfarmgebiete der ostaustralischen Hügelzone. Europas Kernlandschaft finden Ableger in der Poebene, der ungarischen Tiefebene, in Rumänien und Rußland.

Von allgemein eigener, im Detail aber keineswegs einheitlicher Form und Struktur ist die koloniale "Plantagenwirtschaft» im weitesten Sinne.

1. Der im Dienste der Kolonialvölker stehende Landbau der Eingeborenen darf insofern als Plantagenwirtschaft bezeichnet werden, als der Weltmarkt ihr Anbau nach Umfang und Art der Produkte bestimmt. Der Einfluß des weißen Unternehmers und der Wirtschaftspolitik der Staaten ist unverkennbar. Moderne, intensive Anbaumethoden verdrängen immer mehr die extensive, bodenständige Bewirtschaftung. Der Pflugbau, der den Eingeborenen der Tropen unbekannt war - mit Ausnahme Südasiens und Indonesiens -, findet unter Anleitung der Weißen stets größere Verbreitung: so z. B. in Uganda, wo seit 1908 die britische Verwaltung den Baumwollanbau mit energischen Maßnahmen fördert; in Nordnigerien, das 1928 erst drei eingeborene Pflugbauern, 1935 schon deren 600 zählte ${ }^{1 *}$. Zur Hauptsache liefern die Eingeborenen Produkte einjähriger Nutzpflanzen, die keine oder nur eine geringe Aufbereitung erfordern: in Afrika Baumwolle, Erdnüsse (Sudan und Westafrika), Kaffee (Tanganjika), Kakao, Palmkerne und Kolanüsse (Oberguinea); in Asien vorwiegend Reis, Baumwolle und Jute.

2. Die Plantagenwirtschaft im engern Sinne gilt als die koloniale Wirtschaftsform der Weißen. Ihre Betriebe aber sind verschiedener Art. WaIbEL (S. $33 \mathrm{ff}$.) unterscheidet für Afrika nebst dem eigentlichen Plantagenbau eine Farm- und eine Pflanzungswirtschaft. Als Plantage definiert er einen landwirtschaftlichen-industriellen Großbetrieb, der, in der Regel unter Leitung von Europäern, bei großem Aufwand an Arbeit und Kapital hochwertige pflanzliche Produkte für den Weltmarkt erzeugt (Zukker, Tee, Kautschuk, Sisal, Chinarinde) und dabei den Anbau von Nutzpflanzen für den eigenen Bedarf vernachlässigt. Der Pflanzung hingegen fehle der industrielle, großkapitalistische Charakter. Sie beschäftige nur wenige Arbeiter und beschränke sich auf

14 WaIBet, Leo: Die Rohstoffgebiete des tropischen Afrika. Leipzig 1937, S. 296. 
den Anbau von Nutzpflanzen, die leicht aufbereitet und marktfähig gemacht werden können: Kaffee, Tabak, Baumwolle, Bananen u.a.m. Die Farmwirtschaft, als die Betriebsform der Europäer in den gesunden Hochländern der Tropen, begnüge sich mit dem Anbau von Lebensmitteln für den innern Markt.

Diesen besonderen Verhältnissen Rechnung zu tragen, war aus verschiedenen Gründen unmöglich. Der Mangel an Literatur für die außerafrikanischen Tropengebiete und der Generalisierungsgrad der dargestellten Karte verlangten ihre Zusammenfassung. Der in der Legende speziell erwähnte Baumwollgürtel der USA. darf in dieser Hinsicht, trotz den modernen Strukturwandlungen, miteinbezogen werden.

\section{Hinweise zur Interpretation}

Es würde zu weit führen, den Karteninhalt nun auch noch im Sinne der allgemeinen Geographie zu interpretieren. Einige Hinweise jedoch seien gestattet:

1. Der Wert der Karten erschöpft sich keineswegs im bunten Mosaikbild länderkundlichen Details.

Ihre praktische Verwendung im Unterricht wird sicher eine Fülle von Zusammenhängen offenbaren, die zur geographischen Erkenntnis des Wirtschaftslebens der Erde und damit zum Verständnis ihrer mannigfaltigen Wirtschaftslandschaften notwendig sind. Dazu fordern die Karten den Vergleich mit allen möglichen Spezialdarstellungen physischen und kulturellen Belanges: z. B. der Klima- und Bodenkarte, der Produktenund Produktionskarten, Karten der Bevölkerungsdichten, der Völker.

2. So deuten die Karten der Nutzungstypen zum Beispiel in großen Zügen die Bedeutung der physischen, bodenständigen Landschaftseigenschaften für das Bild der Wirtschaftslandschaft. Klima und Vegetationszonen können sich darin widerspiegeln, worauf bei der Besprechung der Legende immer wieder hingewiesen wurde.

Gleiche Naturgrundlagen aber tragen bei weitem nicht immer dieselben wirtschaftlichen Erscheinungen, gleiche Naturlandschaften entwickeln sich keineswegs zu gleichen Wirtschaftslandschaften (Beispiel: Kalifornien und Mittelmeer). Darum ist eine Kritik an den Landbauzonen von ENGELBRECHT und OsBAHR berechtigt. Weil sie den Menschen als Gestalter und Träger der Wirtschaft vernachlässigen, entsprechen ihre Zonen nicht überall den Tatsachen. Der besondere Wert der neuen Karte aber liegt gerade darin, daß sie mittels der Wirtschaftsform die wirtschaftlichen Äußerungen des Menschen in den Vordergrund stellt.

3. So ermöglichen diese Karten - im Vergleich mit den physischen Darstellungen die in der Wirtschaft selbst liegenden Kräfte der wirtschaftslandschaftlichen Gliederung der Erde von jenen zu trennen, die durch die natürlichen Bedingungen gegeben sind.

Diese, den marktorientierten Wirtschaftsformen eigenen Kräfte der Rentabilität die durch die Produktionsbedingungen, durch Angebot und Nachfrage, durch die Verkehrslage gegeben sind - finden auf der Karte sogar unmittelbaren Ausdruck, zum Beispiel in der gürtelartigen Anordnung der Wirtschaftsformen in den Pampas, die an das Thünensche Prinzip erinnert.

4. Ferner erlauben die Karten der Wirtschaftsformen ohne weiteres, die Wirtschaft eines Landes, einer Ländergruppe oder eines Kontinentes nach der Höhe zu beurteilen. Sie vermitteln ein gutes Bild ihrer Wirtschaftsstruktur. Wie ganz anders ist das Bild Afrikas als dasjenige Australiens. Spiegelt sich darin nicht auch die jedem Kontinent eigene wirtschaftsgeschichtliche Entwicklung wieder? Lassen Süd- und Mittelamerika zum Beispiel nicht den altkolonialen Charakter der spanischen Viehwirtschaft erkennen?

Tatsächlich sind die Verwendungs- und Verwertungsmöglichkeiten der neuen wirtschaftsgeographischen Karten groß. Ihre Vielseitigkeit wurzelt darin, daß sie Land- 
schaften als Ganzes zu erfassen versuchen. Mittels der Wirtschaftsformen gelingt es, Týpen von. Wirtschaftslandschaften zu beschreiben. Sie dienen einmal der regionalen länderkundlichen Betrachtung. Zum andern führt ihre räumliche Verteilung auf der Erde zu allgemeinen Erkenntnissen, zu Prinzipien und Gesetzmäßigkeiten.

\section{LES .CARTES ÉĊONOMIQUES}

L'auteur de cet article a remanié les différentes cartes écono mi ques contenues dans cet instrument de travail national qu'est devenu l'A tlas scolaire suisse (enseignement secondaire). Les modifications ont porté sur le contenu et sur la méthode. Bien qu'on se rende compte qu'avec des documents incomplets et disparates, on ne peut que donner une image plus ou moins fidèle d'une période déterminée, on a reporté sur les cartes les principaux changements qui n'étaient pas encore connus lors des dernières revisions de l'atlas (1928 à 1932) et qui caractérisent les dix années précédant la guerre de 1939. Une trop grande limitation dans le choix des matières et dans leur représentation schématique n'est pas désirable, étant donné les diverses exigences correspondant aux différents types d'écoles et de degrés d'âge. C'est aussi pourquoi on n'a pas pu tenir compte, dans la répartition des cartes, d'un programme particulier fixant la disposition des matières. Il n'est pas davantage possible d'adopter une méthode déterminée pour l'ordonnance du contenu. Les cartes analytiques et synthétiques, d'aspect varié, alternent et se complètent, par pays et continents. Toutes ont beaucoup gagné par l'emploi d'une gamme de couleurs judicieusement choisies et par le genre de signatures utilisées. Le principal travail a consisté dans l'établissement de la carte synthétique qui doit représenter certaines unités constituées par toute une série d'éléments distincts. Conformément au but actuel de la géographie économique, qui fait du paysage économique considéré comme un tout, le centre de son étude, on s'est efforcé, pour les cartes principales, d'ordonner l'ensemble de la terre, en types de régions économiques. En corrélation avec les travaux de l'ethnologue HAHN et des géographes économistes Warbel et Whirrleser, dix-huit types ont été établis. D'après la manière et l'intensité selon lesquelles l'homme se procure et utilise les biens matériels et suivant leur degré d'intégration dans l'économie mondiale, ces types peuvent être ordonnés en un système judicieux. $\mathrm{A}$ des formes d'économie extensive viennent s'ajouter des formes intensives; des formes d'économie orientées vers le marché mondial sont présentées face à des formes d'économie autarcique. Sur le plan mondial, les bandes représentant le commerce maritime donnent une image de l'échange des marchandises. Leur largeur correspond à la valeur globale des produits échangés dans les deux sens en 1937. Pour mieux faire comprendre les formes économiques correspondant aux régions économiques représentées, on en décrit, pour terminer, le contenu.

\section{LE CARTE ECONOMICHE}

Tutte le carte economiche contenute nella nuova edizione dell'A tlante per le scuole medie, redatto dal Prof. ED. IмноF, furono eseguite dall'autore del presente lavoro esplicativo. Le variazioni subite dalle stesse sono di natura sostanziale e metodica. Per quanto consapevoli di non poter creare che un quadro approssimativo della situazione economica caratterizzante un'epoca, furono eseguite le revisioni dei dati che non potevano essere noti al momento della stampa dell'ultima edizione (1928 a 1932) e . furono fatte quelle aggiunte che caratterizzano il periodo conclusosi con il 1939. Non sarebbe sicuramente stato desiderabile limitare maggiormente gli argomenti e questo soprattutto se si tengono presenti i diversissimi compiti a cui deve adempiere l'Atlante usato in scuole di tipo e di grado diverso. Soprattutto in relazione a quest'ultimo fatto non è stato possibile seguire un determinato programma, nemmeno una sola linea direttiva nella successione delle carte e tanto meno un unico metodo di rappresentazione della materia. In modo vario, spesso con effetto integrativo, si susseguono perciò le rappresentazioni a nalitiche e sintetiche dei paesi e dei continenti. Esse hanno certamente acquistato in chiarezza grazie ad un'applicazione logica e conseguente della colorazione, delle segnature e dei simboli. La massima cura fu dedicata alla carta globale sintetica che deve rappresentare la distribuzione di particolari unità che alla loro volta sintetizzano tutta una serie di complessi rappresentativi. Nell'intento di corrispondere al criterio essenziale della geografia economica che tratta come argomento principale il paesaggio economico considerato nel suo insieme, nelle carte principali si è voluto dare una visione generale della divisione della Terra in caratteristici paesaggi culturali, che, sulla scorta delle opere dell'etnologo HAHN e dei geografi WAIBEL e W WIrTLESEY, poterono essere riassunti in 18 tipi.

Naturalmente questi paesaggi economici vengono caratterizzati dall'attività umana e a seconda della specie e dell'intensità con cui l'uomo elabora e consuma i prodotti e a seconda dell'importanza che quest'attività assume nell'ambito dell'economia mondiale, i sopraddetti paesaggi si lasciano combinare in un logico sistema generale. Alle economie estensive si associano quelle intensive; a forme economiche producenti beni per il consumo diretto contrastano altre i cui prodotti sono destinati ai mercati interni e mondiale. Una visione generale dello scambio commerciale è reso possibile con striscie rappresentanti le vie marittime che posseggono una larghezza proporzionale alla somma delle merci trasportate in entrambi i sensi durante il 1937. Infine, per permettere una migliore comprensione dei paesaggi distinti, vengono descritte in brevi tratti le attività economiche che caratterizzano i diversi tipi. 